\title{
Quaderni
}

QUADERNI Communication, technologies, pouvoir

73 | Automne 2010

La métropole parisienne entre récits, paroles et échanges

\section{Communication non verbale et avatars au sein d'une communauté virtuelle}

Philippe Bonfils

\author{
(2) OpenEdition \\ Journals \\ Édition électronique \\ URL : http://journals.openedition.org/quaderni/461 \\ DOI : 10.4000/quaderni.461 \\ ISSN : 2105-2956 \\ Éditeur \\ Les éditions de la Maison des sciences de l'Homme \\ Édition imprimée \\ Date de publication : 5 octobre 2010 \\ Pagination : 115-128
}

\section{Référence électronique}

Philippe Bonfils, «Communication non verbale et avatars au sein d'une communauté virtuelle», Quaderni [En ligne], 73 | Automne 2010, mis en ligne le 05 octobre 2012, consulté le 19 avril 2019. URL : http://journals.openedition.org/quaderni/461 ; DOI : 10.4000/quaderni.461 


\section{Technique}

\section{communication}

non verbale

et avatars

au sein d'une

communauté

virtuelle

Le monde de l'enseignement est un acteur clé de l'utilisation des nouvelles technologies de l'information et de la communication. Confronté à la volonté des politiques et à une offre de formation en ligne de plus en plus abondante, ce monde se trouve aujourd'hui à l'intersection de l'industrialisation de la communication et de l'industrialisation de l'éducation (Moeglin, 2005). Cette massification de l'offre nécessite pour autant de s'interroger sur l'utilité, l'efficacité et les limites de dispositifs en ligne chaque jour plus innovants (Proulx, 2004). Si le rôle de la jeunesse est primordial dans l'appropriation et l'usage de ces nouveaux dispositifs (Perriault, 1989), il ne permet pas de cerner correctement les défis didactique, éthiques et communicationnels auxquels les acteurs de l'enseignement doivent se préparer.

De nombreuses études démontrent par exemple les effets d'isolement ressentis par les apprenants à distance, et leurs conséquences en terme d'échecs. L'arrivée de dispositifs de type « mondes virtuels » est à ce titre intéressante. Ces environnements virtuels sont en effets caractérisés par une mise en situation spatiale et métaphorique des

Philippe Bonfils

Enseignant-Chercheur Université du Sud Toulon-Var usages car le monde virtuel simule une certaine forme de réalité. Mais surtout, l'utilisateur pénètre le monde virtuel sous la forme d'un personnage $3 \mathrm{~d}$ (son avatar). Il peut ainsi déambuler à loisir, pour explorer le monde et rencontrer d'autres utilisateurs, en manipulant grâce à son clavier cette figurine interfacée (Dajez, 2006). S'il doit se repérer et maîtriser ce nouveau type d'environnement, il dispose en contrepartie de nouvelles fonctionnalités communicationnelles non verbales liées à son apparence, sa gestuelle et ses mouvements. Ces fonctionnalités lui permettent 
alors une certaine forme d'utilisation proxémique de 1'espace virtuel (Moles \& Rohmer, 1978). Selon nous, cette dimension fortement sociale de l'utilisation de l'espace peut avoir un impact dans des contextes collectifs. Bien que la maîtrise technique et sensorimotrice de ces dispositifs ne soit pas évidente, l'apparition de l'utilisateur sous forme d'individu par l'intermédiaire de son avatar modifie, parasite et enrichie ainsi les interactions sociales et communicationnelles. Nous identifions là une révolution à l'œuvre des usages communicationnels verbaux et non verbaux qui questionne de nombreuses dimensions et médiations (Peraya, 2000) et qui relève d'une approche nouvelle de la communication (Winkin, 2001). Toutefois, partagés entre peurs intrinsèques d'addiction potentielles ou déterminisme technique, la majeure partie des acteurs de l'enseignement n'est pas en mesure aujourd'hui d'apporter de réponses concrètes aux impacts de ces dispositifs dans leur quotidien.

Pour tenter de répondre à ces questions, nous avons mené plusieurs expérimentations grandeur nature entre 2007 et 2009. Nous avons choisi de nous appuyer pour cela sur une pédagogie de projet dans des contextes d'apprentissage collaboratifs (Henri \& Lundgren-Cayrol, 2001). Nous nous sommes notamment intéressés aux processus de construction d'identité des communautés virtuelles et à la création de lien social au sein de groupes collaboratifs à distance. Nous avons fait l'hypothèse que, sous réserve d'une maîtrise suffisante de ces dispositifs, l'irruption de l'avatar par ces modalités nouvelles participait de la création de lien social et renforçait le sentiment de présence à distance dans les groupes d'utilisateurs.

\section{Cadrage théorique}

\section{Dynamique relationnelle et collaboration à distance}

La mise en place croissante de dispositifs d'enseignement à distance suppose la transformation des approches pédagogiques. Celle-ci passe très souvent par la création d'activités collaboratives en groupe qui permettent de renforcer la mise en relation à distance des acteurs. Dans ces approches, Lundgren-Cayrol (2001) souligne que les connaissances sont construites en fonction de l'expérience et du contexte dans lequel elles sont acquises. Elles font souvent l'objet de négociations fortes et d'enjeux de leadership au sein des groupes d'apprenants préalablement constitués. Chaque apprenant doit ainsi partager ses connaissances et mettre au service du groupe ses compétences. Partant de là, il doit donc confronter ses propres schémas mentaux avec ceux des autres membres afin que le groupe puisse se construire une représentation partagée des objectifs, et des solutions apportées collectivement. Nous considérons que ces processus d'acquisition sociale des connaissances sont au cœur des rites d'interactions sociales (Lardellier, 2003). Cette création artificielle de communauté virtuelle d'apprenant par l'institution favorise ainsi l'apparition de micro-cultures dans laquelle les échanges socioaffectifs (Audran \& Daele, 2006) et la solidarité (Papadakis, 2003) occupent une place centrale dans la dynamique relationnelle des acteurs. Des liens forts s'installent et conditionnent l'existence de la communauté virtuelle (Bos-Ciussi, 2006). Ils participent activement de la construction d'identité des groupes par leur fréquence, leur durabilité, et l'engagement émotionnel qu'ils 
génèrent au sein des relations sociales internes. La réussite du groupe passe alors par le partage de valeurs, d'idéaux, d'objectifs et de représentations communes.

L'usage d'internet, et plus récemment des dispositifs de type web 2.0, favorise pour cela l'autonomisation de l'apprenant, l'accès à de multiples sources d'informations et la possibilité pour chaque acteur de contribuer aux dispositifs. À l'inverse, il soulève de nombreuses questions concernant la crédibilité des informations diffusées, et engendre chez certains acteurs une forme courante d'addiction à ces nouveaux outils de communication ludiques et instantanés : " l'humain qui se sert de ces machines est libre et joyeux. Il n'a jamais de mauvaises pensées contre les autres, ni contre lui-même, puisqu'il est toujours logé dans l'efficacité de l'instant communicant »(Sfez, 2007).

\section{L'avatar représentant des individus de la com- munauté virtuelle}

Les mondes virtuels se révèlent quant à eux des espaces complexes de représentation car ils sont déterritorialisés et ont des temporalités multiples. Comme dans tout espace virtuel traditionnel (type web 2.0), tout utilisateur interagit de manière synchrone dans des lieux physiques différents et selon des décalages horaires potentiels : il y contraction de l'espace-temps.

Au delà de cet aspect, l'irruption de l'avatar a un impact direct sur la représentation car elle engendre de nouveaux rapports à la présence pour les utilisateurs. Désormais représentés sous forme d'individus par l'entremise de leur avatar, les uti- lisateurs deviennent des spectateur-acteurs de ces nouveaux espaces : il y déplacement d'existence (Weissberg, 1999) et dès lors nécessité d'intégrer cette nouvelle variable dans l'analyse. Il y a non seulement la représentation d'une présence psychique mais aussi la représentation d'une présence physique dans le même espace-temps: « et cette question est d'une grande complexité dès qu'on refuse d'identifier présence corporelle et présence psychique et qu'on dissocie l'unité de lieu et de temps dans la multiplicité des espaces-temps mentaux » (Weissberg, 1999, p. 5). L'utilisateur peut désormais exister aussi à l'écran par l'intermédiaire de sa projection-avatar, son « faux-double » (Turkle, 1997).

L'avatar, par ces nouvelles modalités communicationnelles non verbales, enrichit et parasite aussi l'espace virtuel de significations partagées. Par cette mise en scène possible des acteurs du dispositif, il favorise aussi la perception d'une réalité simulée, il participe à la dynamique relationnelle de groupe et crée du lien social.

\section{Communication non verbale et nouvelle com- munication}

Comme évoqué plus haut, nous avons adopté pour l'étude de notre objet de recherche une approche « nouvelle communication » (Winkin, 2001). Cette nouvelle vision de la communication est issue du courant de l'école de Palo Alto. Elle a été amorcée par Bateson et suivie par de nombreux chercheurs issus pour beaucoup des thérapies systémiques (Jackson, Watzlawick), de la sociologie, de la psychologie et de l'anthropologie (Goffman, Hall, Birdwisthell). Regroupés dans ce que l'on a nommé le Collège invisible ${ }^{l}$, ces chercheurs ont 
adhéré à cette approche qui associe aux modèles traditionnels de la communication tous les codes qui participent de constructions de significations et plus largement de la communication. Au delà d'une prise en compte systémique de ces codes, cette approche considère que la communication est un processus social complexe et permanent qui donne lieu à de multiples comportements symboliques. Elle tient par exemple compte dans l'analyse de la parole, des mimiques, du geste, des postures et des situations dans l'espace interindividuel. Cette approche fait donc l'hypothèse que tout acteur d'une situation de communication élabore consciemment ou inconsciemment un système de significations sur plusieurs canaux à la fois. Elle s'apparente aussi à une forme de sémiopragmatique de la communication et constitue aujourd'hui un des paradigmes de l'analyse des processus de communication.

Inscrit dans cette approche, nous considérons ainsi dans notre étude que l'avatar peut être considéré comme un support potentiel de proxémique. Celui-ci autorise en effet des déplacements, des postures, et de la gestuelle qui font tous partie de l'analyse des codes non verbaux. Il y a donc lieu de les considérer comme des indicateurs de formes variées de communication.

\section{Les rites d'interactions et la construction de lien social}

L'espace virtuel peut, selon les usages et les modalités, être interprété comme un espace privé ou public. Il peut donc donner lieu pour les acteurs à des comportements individuels et collectifs dans ces deux dimensions. Dans les mondes virtuels, l'apparition de l'individu par la présence de l'ava- tar peut renforcer ces aspects en autorisant divers types de mouvements allant jusqu'à des comportements carnavalesques. Protégé par le masqueécran que constitue l'avatar, l'utilisateur-acteur peut préserver sa propre image (Goffman, 1974), tout en adoptant des comportements libérés des contraintes de la communication propre aux contextes physiques et présentiels.

Nous verrons plus loin dans notre expérimentation que notre échantillon est constitué d'apprenants à distance, préalablement constitués en groupes, et régulièrement rassemblés avec un tuteur représentant de l'institution pédagogique. Ces situations récurrentes s'apparentent pour nous à des sphères courantes de personnes privées (groupes d'apprenants) rassemblées en un public (l'institution), ce que Habermas qualifie de sphère publique bourgeoise où règne l'usage public de la raison (Habermas, 1978).

Ainsi, même si l'environnement virtuel offre des caractéristiques sensori-motrices (déambulation, vol, téléportation, postures, gestuelles) et symboliques (caractérisation de l'avatar, personnalisation de l'environnement) qui sont ludiques et conviviales, il doit permettre de réguler socialement les comportements sur le modèle d'une situation physique et présentielle basée sur la raison. Partagés entre situation formelle et informelle, les utilisateurs doivent donc pouvoir adopter des rites d'interactions qui permettent au groupe de collaborer et donc de communiquer en limitant les dispersions.

\section{Modalités de l'expérimentation}

Notre expérimentation s'est déroulée à l'Ufr 
en Sciences de l'information et communication de l'Université du Sud Toulon-Var : L'institut Ingémédia ${ }^{2}$. Un panel d'étudiants a été constitué au sein de la promotion de licences professionnelles TAIS 2007 (80 étudiants constitués en 12 groupes). Les étudiants étaient alors en situation de projet de création d'une campagne de communication multi-supports. Dans ce projet les étudiants devaient mettre en œuvre les enseignements théoriques de management de projet et développer une culture collaborative par une mise en situation concrète. Ils devaient aussi utiliser tout un ensemble d'outils de communication à distance ainsi qu'une plateforme collaborative.

L'expérimentation a consisté à mettre à leur disposition un monde virtuel à mi-parcours de leur projet. Ils disposaient alors d'un bâtiment virtuel avec une salle par groupe dans le dispositif Second Life ${ }^{3}$, et avaient pour objectifs d'y organiser des réunions de suivi de projet à distance avec un tuteur. Les étudiants ont dû commencer par s'enregistrer dans le dispositif et installer le navigateur 3D semi-immersif I $^{4}$ Ils ont pu ensuite caractériser leur avatar, puis commencer à mâ̂triser leur insertion corporelle dans l'environnement spatialisé. Cette première phase leur a ainsi permis d'explorer l'univers virtuel grâce à des fonctions naturelles de déplacement comme la marche, mais aussi à des fonctions plus originales comme le vol ou la téléportation ${ }^{5}$ instantanée d'un lieu à un autre. La seconde phase a consisté pour les étudiants à organiser leur première réunion (séance du 03/04/07) dans une salle virtuelle anonyme (modalités libres). Elle a donné lieu aux premières pratiques communicationnelles collectives (chat, voix sur IP, gestuelle, mouvements et déplacements). La deuxième réunion (séance du 11/04/07) a porté sur la personnalisation de leur propre salle virtuelle amenant les étudiants à partager divers type d'informations (objets $3 \mathrm{D}$, contenus divers type images et vidéos). Les troisième (séance du 03/05/07) et quatrième réunions (séance du 09/05/07) ont directement porté sur le suivi du projet (les étudiants manipulant à loisir toutes les fonctionnalités du dispositif). Au final, durant la période de mars à mai 2007, chaque groupe a organisé une réunion tous les quinze jours au sein du monde virtuel. Chaque réunion s'est déroulée sur une durée moyenne de 30 minutes.

Sur le plan méthodologique, les résultats présentés dans cet article sont issus d'un corpus de données récoltées pendant les réunions des étudiants avec le tuteur. Le corpus est structuré en captures d'écrans et en films de captation vidéo des séances. Différentes grilles de dépouillement ont été constituées avec des modalités spécifiques selon les variables et indicateurs étudiés (zones proxémiques, proximités, postures, gestuelles). Dans la mesure où certaines variables ont été partiellement manipulées (situation, groupe, dispositif, contenus des réunions), l'approche peut être considérée comme expérimentale. Elle est aussi compréhensive car nous avons procédé à une analyse qualitative par re-thématisation des résultats, en croisant ceux-ci avec certains résultats de questionnaires distribués après l'expérimentation (Mucchielli \& Paillé, 2005).

\section{Résultats}

\section{Usages proxémiques}

La première variable proxémique étudiée porte 
sur les modalités d'utilisation de l'espace virtuel par les utilisateurs et ceci en manipulant leur avatar. Cette variable est livrée ici avec trois indicateurs pour l'analyse : la situation des avatars, l'évolution de la proximité des avatars et l'évolution des postures des avatars dans l'espace commun. Il s'agit ici d'identifier d'éventuelles récurrences dans ces modalités sensori-motrices et spatiales, et partant de là de pré-valider des usages communicationnels non verbaux.

\section{Situation des avatars dans l'espace commun :}

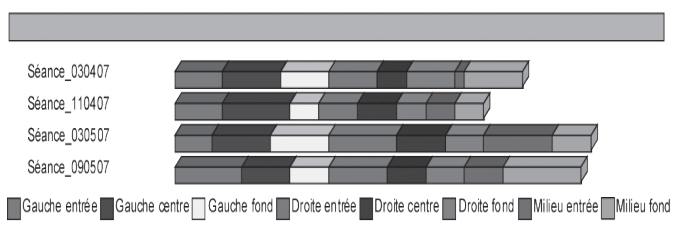

Figure 1: Situation des avatars dans les zones de l'espace commun

Ce premier indicateur est construit sur l'étude de l'occupation des zones proxémiques des avatars durant les réunions avec le tuteur. La grille est constituée de huit modalités correspondantes à un découpage de l'espace virtuel en huit zones (côtés gauches, centraux et droits de la salle). Les résultats montrent une occupation assez régulière de tous les espaces dans la salle, et ce quelles que soient les caractéristiques de l'échantillon (âge, cursus, genre, pays d'origine, etc.). La multiplicité des modalités nous amène à considérer ces résultats comme des tendances. Toutefois, grâce à l'étude des visuels enregistrés pendant les séances (voir extraits en annexes), nous constatons qu'une majeure partie des groupes a meublé la salle de réunion avec une table située au centre de celleci. Cet ameublement est suffisamment significatif pour que l'on suggère qu'il a sans doute eu une influence sur l'usage des zones proxémiques par les avatars (voies de circulations limitées ou induites).

\section{Évolution de la proximité des avatars}

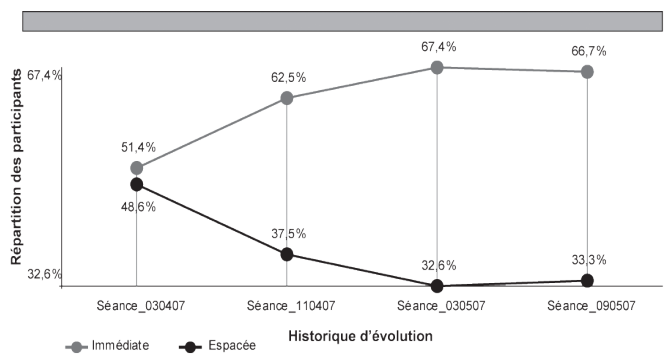

Figure 2: Évolution de la proximité des avatars

Le deuxième indicateur proposé porte sur l'étude de l'évolution de la proximité des avatars. Si la présence d'une table au centre a induit des usages proxémiques spécifiques, cet indicateur est susceptible de nous renseigner plus finement sur le niveau de proximité et de distance interpersonnelles des avatars. La grille d'analyse est construite pour cet indicateur selon deux modalités de proximité immédiate ou espacée (place libres entre avatar). Au terme des quatre séances, nous constatons une nette évolution des proximités des sujets présents $(86,7 \%)$. Si les utilisateurs situent majoritairement leurs avatars autour de la table au centre de la salle, nous notons aussi de manière significative qu'ils laissent de moins en moins d'espaces vides entre les avatars.

Ce dernier indicateur proxémique permet d'étudier l'évolution des postures des avatars. Nous essayons de déterminer si les avatars utilisent des objets aussi usuels que les sièges pour participer aux réunions assis. La grille est construite selon deux modalités simples de posture (debout ou 
assis). Les résultats montrent plusieurs phases de postures dans les groupes. Au début de l'expérimentation, la plupart des utilisateurs maintiennent leur avatar assis (94,7\%). De la deuxième à la troisième réunion, un nombre plus important d'utilisateurs laissent leur avatar debout (78,6\% et $79,1 \%$ ). Cette tendance diminue jusqu'à la dernière séance avec une majorité d'avatars assis $(86,7 \%)$.

\section{Évolution des postures des avatars:}

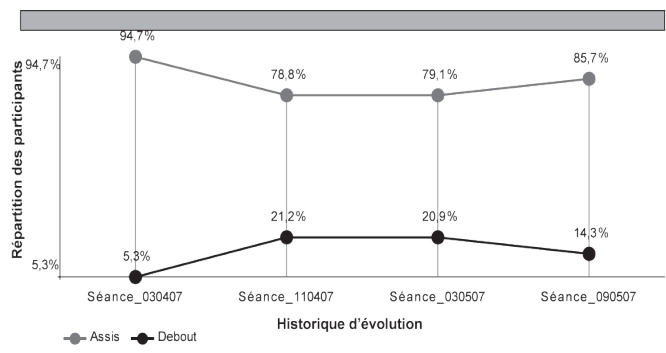

Figure 3: Évolution des postures des avatars

\section{Usages kinésiques}

En second lieu, nous livrons une variable kinésique susceptible de nous renseigner sur les modalités d'usage de la gestuelle des avatars par les utilisateurs. Cette variable s'appuie sur deux indicateurs principaux : L'analyse de l'évolution de la récurrence de la gestuelle des avatars et des causes d'utilisation de la gestuelle des avatars. Nous souhaitons démontrer ici que la gestuelle participe elle aussi, par l'intermédiaire des avatars, de la communication non verbale des groupes.

L'indicateur d'évolution de récurrence de la gestuelle des avatars apparaît comme assez faible durant l'expérimentation. La grille d'analyse est structurée autour de quatre modalités (utilisation nulle, rare, régulière et très régulière de la gestuelle). Lors de la première séance, $40 \%$ des étudiants découvrent et expérimentent ces fonctionnalités kinésiques (et ce de manière équivalente entre utilisation rare et régulière). Ce chiffre baisse lors de la deuxième séance pour passer à 10\% (uniquement sur une utilisation rare). Si le volume croît à nouveau à la troisième séance, nous constatons surtout que la gestuelle est régulièrement adoptée par ceux qui l'ont testée après la deuxième séance. Nous identifions des usages très réguliers pour $7 \%$ des sujets $(9 \%$ rarement et $9 \%$ régulièrement). La dernière séance montre enfin un nouvel accroissement et une stabilisation de la gestuelle régulière pour $30 \%$ des sujets avec une répartition homogène ( $15 \%$ rarement, et $15 \%$ régulièrement).

\section{Évolution de la récurrence de la gestuelle des} avatars:

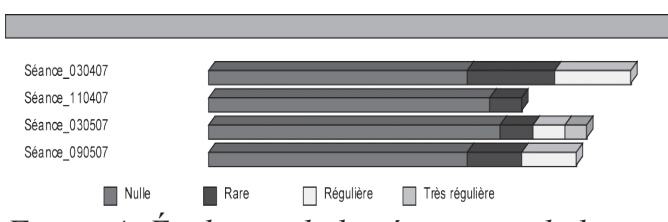

Figure 4: Évolution de la récurrence de la gestuelle des avatars

Évolution des causes d'utilisation de la gestuelle des avatars:

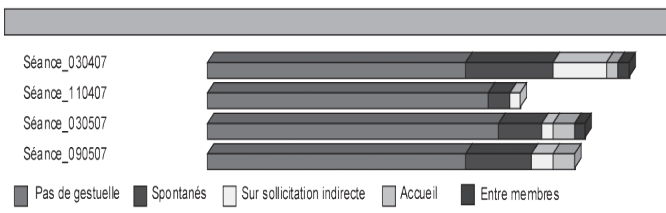

Figure 5: Évolution des causes d'utilisation de la gestuelle 
Ce dernier indicateur de gestuelle nous permet d'étudier l'évolution des causes d'utilisation de la gestuelle des avatars. La grille est cette fois-ci bâtie autour de cinq modalités (pas de gestuelle, gestuelle spontanée, en réponse à une sollicitation indirecte, comme geste d'accueil, ou lors d'échanges entre membres). Le cycle d'évolution correspond au cycle de récurrence : un intérêt certain en début d'expérimentation $(35,9 \%$ des sujets), suivi d'une baisse en cours d'expérimentation ( $10,3 \%$ de sujets), puis d'une croissance ( $22,9 \%$ des sujets) et enfin une stabilisation en fin d'expérimentation $(29,4 \%)$. La première séance montre une répartition équitable entre gestuelle spontanée et sur sollicitation indirecte pour $40 \%$ des sujets (la gestuelle d'accueil et entre membres étant marginale). La deuxième séance montre un tassement important des usages (10\%). En revanche, les troisième et dernière séances montrent le retour significatif de la gestuelle spontanée $(12 \%$ puis $18 \%$ ), et une stabilisation de la gestuelle sur sollicitation indirecte ( $3 \%$ puis $6 \%$ ) et d'accueil (6\% puis $6 \%)$.

\section{Discussions}

\section{Récits commentés des séances}

L'expérimentation a donné lieu à une première phase d'acculturation technique et de maîtrise du dispositif. Les mondes virtuels sont en effet généralement accessibles à partir de navigateurs spécifiques très riches en fonctionnalités et donc aussi parfois complexes à maitriser. Cette phase a malgré tout été assez courte car l'échantillon représentatif d'étudiants avait déjà utilisé des dispositifs similaires en images de synthèse. Pour la plupart, les étudiants étaient utilisateurs d'en- vironnements ludiques de type jeu vidéo en ligne (type World of Warcraft ${ }^{\circ}$ ). Durant l'expérimentation, les problèmes les plus courants rencontrés par les utilisateurs concernaient la gestion des points de vue (difficultés à se repérer au mieux dans un environnement spatial). Ceux-ci devaient en effet recomposer leurs schèmes sensori-moteurs suivant une logique artefactuelle d'instrumentation (Rabardel, 1995), non seulement pour apprendre à se situer dans l'espace, mais aussi pour manipuler leur avatar. Ces problèmes ont diminué rapidement et ont été surmontés pour la majeure partie des sujets dès la deuxième séance.

Ces lors de ces deux premières séances que les utilisateurs ou sujets ont appris à investir le monde virtuel, et à caractériser leur avatar avec plus ou moins de ressemblance ou d'opacité par rapport à leur physique réel. C'est à ce moment là que l'effet de masque a joué à plein (Goffman, 1974) grâce à la modification constamment possible et plastique du visage, du corps, des vêtements et des accessoires du masque ou sosie (Auray, 2004). C'était aussi l'occasion pour eux de tester les mouvements, les postures et les gestes de leur avatar, et donc de créer de nouvelles modalités communicationnelles (l'espace 3D présente en effet des caractéristiques créatives fortes et engendre souvent des situations ludiques). Les échanges furent de plus en plus nourris jusqu'à la troisième phase qui a amené les acteurs du dispositif à partager des ressources 3D (type objets interactifs, photos, vidéos, liens vers des sites, scripts) et à échanger sur les objectifs de leur projet. À ce stade nous avons constaté le règne des experts techniques qui maîtrisaient parfaitement le dispositif et qui contribuaient fortement en objets $3 \mathrm{D}$ et en ressources. La 
communication non verbale s'est alors installée dans les groupes et l'on a vu émerger peu à peu des rites d'interactions qui s'appuyaient de plus en plus sur de la communication non verbale. Les rencontres se sont structurées très souvent autour d'un rituel qui ressemblait aux situations similaires dans des environnements physiques. Les utilisateurs pénétraient par l'intermédiaire de leur avatar dans la salle de la réunion et choisissaient une place autour de la table virtuelle. On assistait généralement à un accueil manifesté par des gestes de salut, et à de brefs échanges pour valider les positions de chacun (figure 1). Les places étaient de plus en plus ritualisées et les extrémités des tables de forme orthogonales étaient le plus souvent réservées aux responsables de groupe ou au tuteur. Le reste du groupe occupait alors les espaces restants de manière plus spontanée. Au fur et à mesure des séances les utilisateurs laissaient de moins en moins d'espaces vides entre eux (figure 2). ils restaient la plupart du temps assis (figure 3) comme pour reproduire des micro-rites de politesse entre sphère intime et sociale.

La suite des échanges entre utilisateurs était répartie entre usages digitaux $\left(\mathrm{chat}^{7}\right)$ et utilisation plus ou moins récurrente de la gestuelle (figure 4) pour réagir spontanément ou non aux interventions de chacun et parfois ponctuer des décisions (figure 5).

\section{Analyse des résultats}

Grâce aux résultats de questionnaires postérieurs à l'expérimentation, nous savons que pour une très grande partie des sujets l'avatar est considéré comme une création imaginaire qui ne ressemble pas forcément à l'utilisateur. Ce niveau de ressemblance apparaît couplé à la typologie de la situation interpersonnelle (en raison des modalités symboliques particulières des mondes virtuels, celle-ci varie de mi-formelle à mi-informelle). Nous suggérons alors que l'opacité de l'avatarmasque agit comme facteur d'équilibre social pour réguler les situations et permet à chacun de s'adapter au fonctionnement en micro-culture (Audran \& Daele, 2006). Le masque devient ainsi libérateur et autorise certaines libertés d'expression sur des registres socio-affectifs. Il permet à divers sujets de s'exprimer là où ils ne se seraient probablement pas manifestés dans les mêmes situations interpersonnelles en face à face. Dans certains cas, nous soulignons que l'effet masque peut aussi brouiller les pistes situationnelles car il transforme les limites comportementales, et rend poreuses les frontières identitaires. La multiplication des niveaux de masque peut aller par exemple pour certains utilisateurs jusqu'à la construction de plusieurs projections de soi numériques (Mabillot, 2000). D'éventuelles addictions à cette nouvelle forme de liberté psychique sont alors possibles. Quels que soient les usages de l'avatar sur le plan de la caractérisation, comment ne pas imaginer dès lors que cela n'ait pas d'impact sur la construction d'identité et le lien social dans un groupe ?

Ces résultats sur la caractérisation de l'avatar sont enrichis par l'analyse des variables proxémiques et kinésiques au cœur de cet article. En effet, si l'avatar est un support potentiel de personnalisation de l'utilisateur en terme d'apparence, il est aussi l'acteur qui permet de transférer virtuellement des attitudes et des comportements physiques et corporel. 
Ainsi, les modalités d'appropriation de l'espace et de la gestuelle renforcent de notre point de vue ces effets. Elles peuvent à ce titre offrir une nouvelle dimension d'échange dès qu'elles répondent à un rituel spontanément acté par tous les acteurs du dispositif. Nous pensons, comme Lardellier (2005), que ce lien social peut prendre plusieurs formes y compris non verbales : symboliques à travers les interactions, techniques par le jeu des médiatisations et médiations, discursives et énonciatives du point de vue sémiologique et sémio-pragmatique. Nos résultats montrent ainsi l'apparition progressive d'un rituel social d'accueil et de démarrage des réunions par le salut gestuel, l'installation autour d'une table, et la limitation des places vides. Cette gestuelle revient à des moments clés de discussion et de résolution de conflits et apparaît dans le processus de régulation communicationnelle du groupe. Maîtrisée par les sujets, elle met alors en exergue des comportements communicationnels stabilisés et partagés qui font office de médiation sensorimotrice, sociale et praxéologique (Peraya, 2007), et agissent comme des artefacts communicationnels au sein des groupes (Agostinelli, 2003). L'avatar permet alors non seulement de « saisir » des objets virtuels, mais il permet aussi d'installer une relation corporelle et « d'occuper » l'espace virtuel. De notre point de vue, il fait sens dans le groupe sur le plan d'une approche anthropologique de type nouvelle communication (Winkin, 2001).

Suivant Hall (1971), nous notons aussi que les interactions spatiales s'inscrivent dans des espaces proxémiques entre distance personnelle lointaine (de 75 à $125 \mathrm{~cm}$ ) et distance sociale proche (de $1,20 \mathrm{~m}$ à $2,10 \mathrm{~m}$ ). Ces zones proxémiques territo- rialisées et associées à la pratique de la gestuelle, nous renseignent sur un niveau d'intimité qui relève de la sphère privée, de la convivialité et d'une certaine forme poétique de l'espace : " saisi par l'imagination, il ne peut pas rester l'espace indifférent, il est vécu » (Bachelard, 1957). Réincarné par l'avatar, le sujet manipule alors l'espace virtuel et se l'approprie en fonction du contexte qui perçoit. La disparition des déambulations parasites et des stations debout pendant les réunions (figure 2 et 3 ) suggère aussi cette adoption d'un rituel social et communicationnel (Goffman, 1974). Les sujets se comportent alors conformément à des normes sociales très proches de situations physiques similaires : «ils se conforment aux actes que l'on attend d'eux dans la vie réelle » (Castronova, 2003).

Plus encore, le sujet par sa condition nouvelle de spectateur-acteur a une perception nouvelle de la communication. Non seulement, grâce à l'image de synthèse, la situation est désormais matérialisée sous ses yeux, mais dans le même temps comme dans un miroir il se voit agir dans cette situation de communication concrète à laquelle il participe (Bonfils, 2007). Il dispose de plusieurs points de vue sur cette situation qui peuvent à la fois le renseigner sur les actes communicationnels du groupe, et aussi de manière plus inédite sur ses propres actes communicationnels (effet de miroir). Cette situation peut libérer certains sujets et dans le même temps en déstabiliser et inhiber d'autres qui choisissent de se manifestent uniquement par les canaux traditionnels de communication. Nous voyons là une des raisons du faible taux d'utilisation de la gestuelle. Ceci est renforcé par le fait que la manipulation de l'avatar nécessite l'utilisation du clavier. 


\section{Conclusion}

Nos résultats démontrent la complexité des variables étudiées car ils montrent que de nombreux facteurs peuvent conditionner la manipulation de l'avatar à des fins communicationnelles. Nous avons souligné l'impact des médiations sensori -motrices dans les mondes virtuels, et partant de là, la nécessité pour les sujets de maîtriser des interfaces souvent très riches. Nous avons pu constater l'émergence d'usages communicationnels non verbaux nouveaux même si nous ne pouvons pas conclure sur une adhésion massive. Les raisons sont nombreuses (techniques, sensori-motrices, culturelles), mais pour autant nos résultats démontrent la potentialité de l'avatar comme support d'utilisation proxémique et kinésique d'un espace numérique métaphorique et spatialisé. Notre expérimentation nous a aussi permis de valider plusieurs hypothèses concernant l'impact de l'avatar sur l'émergence de rituels et la création de lien social au sein de communautés virtuelles.

Pour conclure, nous assistons au développement en cours d'une nouvelle génération de périphériques qui sont associés à de nouvelles modalités ergonomiques (écrans tactiles des Iphone et des Pc, capteurs sans fils, manettes type Wii, etc.). Nous faisons l'hypothèse que l'utilisation de la gestuelle va être grandement facilitée et engendrera des espaces virtuels qui se transformeront graduellement pour devenir des espaces de représentation et de significations partagées (Bougnoux, 2006). Nous imaginons dès lors que ces espaces se prêteront à des expériences de mises en situation et dans certains cas de théâtralisation de la communication. La piste des Serious game semble par exemple prometteuse. Nous pensons qu'elle permettra de scénariser divers types de situations et d'activités dans lesquels l'espace et la gestuelle seront nécessaires au bon déroulement des actions et aux résolutions des problèmes posés. Un vaste champ de recherche s'ouvre donc qui ira de l'apprentissage au jeu collectif, et où le jeu de rôle et la convivialité occuperont une place indéniable. 


\section{$R \cdot E ́ \cdot F \cdot \dot{E} \cdot R \cdot E \cdot N \cdot C \cdot E \cdot S$}

AGOSTINELLI, S. (2003). Les nouveaux outils de communication des savoirs. Éd. L'Harmattan (Collection Communication et Civilisation), Paris. 210 pages.

AUDRAN, J., \& DAELE, A. (2006). Forums et liste de diffusion : rapport à la communauté et «micro-culture». À paraître.

AURAY, N. (2004). Sosies et avatars dans les jeux : entre écriture et image. In L'image sosie (pp. 95-107). $1^{\text {er }}$ colloque international IcôneImage. Musée de Sens. France.

BATESON, G. (1977). Vers une écologie de l'esprit. Tome 1. Éd. Seuil, Paris. 281 pages.

BIRDWHISTELL, R.L. (1968). Kinesics. In Sills (David L.), Éd International Encyclopedia of the Social sciences, New York, Mac Millan, vol. 8, pp. 379-385

BONFILS, P. (2007). Dispositifs socio-techniques et mondes persistants : Quelles médiations pour quelle communication dans un contexte situé ? Thèse de doctorat. Université du sud Toulon-Var. France.

BOUGNOUX, D. (2006). La crise de la représentation. Éd. La Découverte, Paris. 183 pages.

CASTRONOVA, E. (2003). « Theory of the avatar ». CESifo working papers $n^{\circ} 863$. Category 2 : public choice.

DAJEZ, F. (2006). « La figurine interfaçée à propos de l'odyssée d'Abe ». In BARBOZA, P., \& WEISSBERG, J. (2006). L'image-actée. Éd. L'Harmattan, Paris. 270 pages.

GOFFMAN, E. (1974). Les rites d'interactions. Les éditions de Minuit, Paris. 230 pages.

HABERMAS, J. (1978). L’Espace public. Éd Payot, Paris. 324 pages.
HALL, E.T. (1971). La dimension cachée. Ed. Seuil (Collection Points essais), Paris. 254 pages. Henri \& Lundgren-Cayrol

LARDELLIER, P. (2003). Théorie du lien rituel. Ed L'Harmattan. 237 pages.

LARDELLIER, P. (2005). « Report : Formes Techniques, Formes Sociales ». In : TRANS. Internet Zeitschrift für Kulturwissenschaften. No. 16/2005.

MABILLOT, V. (2000). Mises en scène de l'interactivité. Thèse de doctorat, Université de Lyon II. France.

MUCCHIELLI, A., \& PAILLÉ, P. (2005). L'analyse qualitative en sciences humaines et sociales. Éd. Armand Collin. 211 pages.

MOEGLIN, P. (2005). Outils et médias éducatifs, une approche communicationnelle. Éd. Presses Universitaires de Grenoble. 296 pages.

MOLES, A., \& ROHMER, I. (1978). La psychologie de l'espace, $2^{e}$ Éd. Casterman, Paris.

PAPADAKIS, M.C (2003). People Can Create a Sense of Community in Cyberspace, SRI International, Arlington, VA (USA).

PERRIAULT, J. (1989). La logique de l'usage. Essai sur les machines à communiquer. Éd. Flammarion, Paris. 254 pages.

PROULX, S. (2004). « Les communautés virtuelles construisent-elles du lien social?». Communication, Colloque international «L'organisation média. Dispositifs médiatiques, sémiotiques et de médiations de l'organisation ». Université Jean Moulin, Lyon, 19-20 novembre 2004.

PERAYA, D (2007). « Les dispositifs pédagogiques médiatisés : l'articulation entre médiation et médiatisation ». Université Technique de Compiègne. Groupe de recherche ASSUN.

PERAYA, D. (2000). " Internet : un nouveau dispositif de médiation des savoirs et des com- 
portements ». In Journées d'études. Eduquer aux médias à l'heure du multimédia, Conseil de l'Éducation aux médias. Bruxelles.

RABARDEL, P. (1995). Les Hommes et les technologies une approche cognitive des instruments contemporains. Paris : Université de Paris 8.

SFEZ, L. (2007). "Les futurs de la communication". Revistas Eletrônicas de Ciências da Comunicação, Vol.5.

TURKLE, S. (1997). Life on screen. Ed Simon and Schuster, New York. 352 pages.

WEISSBERG, J.L. (1999). Présence à distance. Déplacements virtuels et réseaux numériques ; Pourquoi nous ne croyons plus à la télévision. Éd. L'Harmattan, Paris. 304 pages.

WINKIN, Y. (2001). Anthropologie de la communication. $2^{\mathrm{e}}$ Éd. Seuil (collection Points-Essais), Paris. 288 pages.

WINKIN, Y. (1981). La nouvelle communication. $3^{\text {e }}$ Éd. Seuil (collection Points-Essais), Paris, 2000. 390 pages.

EXEMPLES DE VISUELS DU DISPOSITIF (Bonfils, 2007)

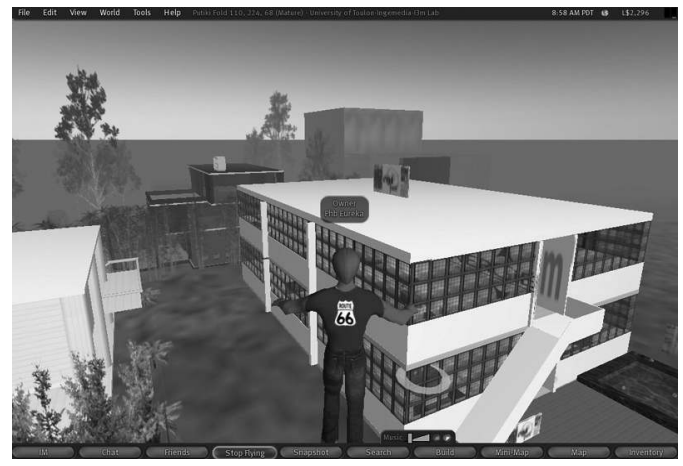

Figure 6: Vue générale du bâtiment virtuel de l'institut Ingémédia (première Ufr française présente au sein du monde virtuel Second Life)

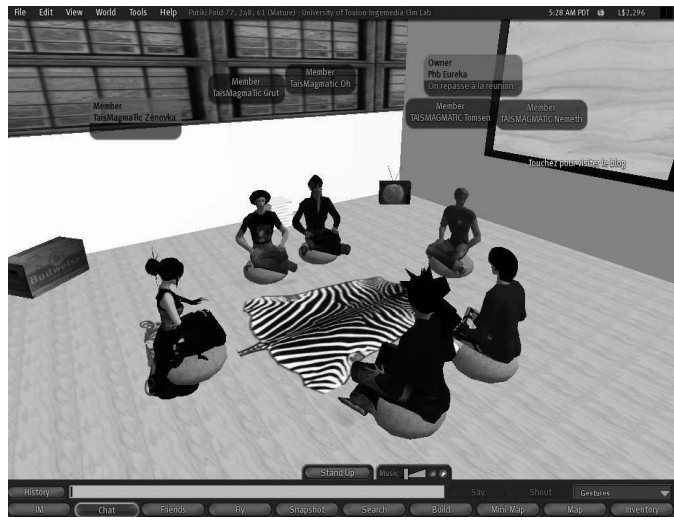

Figure 7: Capture écran d'une séance de cours à l'intérieur du bâtiment virtuel

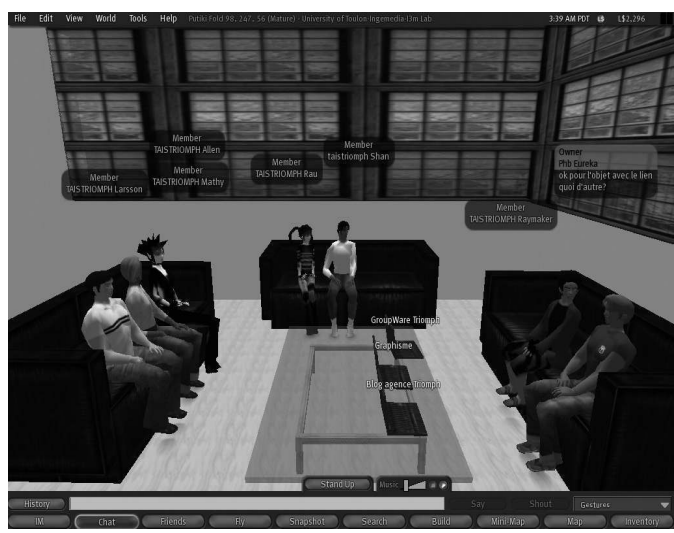

Figure 8: Une salle virtuelle de réunion meublée d'objets interactifs 


\section{$\mathrm{N} \cdot \mathrm{O} \cdot \mathrm{T} \cdot \mathrm{E} \cdot \mathrm{S} \mathrm{R} \cdot \mathrm{E} \cdot \mathrm{S} \cdot \mathrm{U} \cdot \mathrm{M} \cdot \mathrm{E}$}

1. Chercheurs (Gregory Bateson, Erwin Goffman, Ray Birdwhistel, Edward T. Hall) qui, à partir des années 1950, s'appuyant sur les concepts issus de la cybernétique et de la théorie générale des systèmes, proposèrent une vision des phénomènes de communication en rupture avec le traditionnel modèle « télégraphique» : un émetteur adresse un message à un récepteur qui le décode (http://www.scienceshumaines.com/).

2. Voir http://www.ingemedia.net

3. Voir http://www.secondlife.com

4. Le dispositif est considéré comme semi-immersif car il ne nécessite pas de périphériques haptiques lourds comme des casques de vision, des caméras de tracking ou des gants magnétiques de manipulation.

5. On nomme téléportation le transfert d'un corps dans l'espace sans parcours physique des points intermédiaires entre départ et arrivée. Le thème vaut pour la science-fiction, la physique ou la parapsychologie (http://www.techno-science.net/).

6. World of Warcraft est un jeu massivement multijoueurs (MMORPG) qui permet à des milliers de joueurs de se rencontrer en ligne et de s'allier contre l'univers du jeu ou se combattre entre eux. Ce jeu compte actuellement près de 15 millions de joueurs de par le monde. Il est édité par la société Blizzard (http:// www.wow-europe.com).

7. La fonction « voix » n'étant pas disponible lors de cette expérimentation, nous n'avons pas pu tester les modalités de communication verbale directe.
Cet article s'intéresse aux enjeux théoriques des nouvelles modalités communicationnelles offertes par des dispositifs de type « mondes ou univers virtuels». Dans ces dispositifs récents, il est en effet possible pour l'utilisateur d'une communauté virtuelle de naviguer et de communiquer à distance au sein d'un environnement métaphorique et spatial. L'utilisateur dispose alors d'objets 3D et d'une représentation de lui même qui prend la forme d'un personnage que l'on nomme couramment son « avatar ». Il s'agit pour nous ici de réfléchir aux conséquences éthiques, sociales et communicationnelles de la matérialisation en trois dimensions de l'utilisateur dans un espace virtuel. Cet espace met alors en scène une situation de communication dans laquelle la représentation est omniprésente. Sur le plan épistémologique, nous situons cet objet de recherche au carrefour d'une approche de type nouvelle communication (Winkin, 2001), et de l'analyse sémiopragmatique de médiations sensorimotrices et sociales (Peraya, 2007). Afin de préciser notre réflexion, nous livrons au lecteur les résultats d'une expérimentation menée dans une communauté virtuelle d'apprenants (Bonfils, 2007). Grâce à la manipulation de son avatar, nous montrons comment chaque membre de la communauté utilise la communication non verbale et tend à participer à la dynamique relationnelle et à la construction d'un lien social collectif. Nous démontrons que par l'intermédiaire d'une ritualisation progressive des mouvements et des interactions des avatars (Goffman, 1973 ; Lardellier, 2003), l'espace virtuel apparaît comme un lieu symbolique et proxémique de communication (Hall, 1971). 